\title{
A rapid approach to investigate spatiotemporal distribution of phytohormones in rice
}

\author{
Wen-Jing Cai ${ }^{\dagger}$, Tian-Tian Ye ${ }^{\dagger}$, Qing Wang, Bao-Dong Cai and Yu-Qi Feng ${ }^{*}$
}

\begin{abstract}
Background: Phytohormones play crucial roles in almost all stages of plant growth and development. Accurate and simultaneous determination of multiple phytohormones enabled us to better understand the physiological functions and the regulatory networks of phytohormones. However, simultaneous determination of multiple phytohormones in plant is still a challenge due to their low concentrations, structural and chemical diversity, and complex matrix of plant tissues. Therefore, development of a simple and selective method for the simultaneous determination of multiple phytohormones is highly needed.
\end{abstract}

Results: We developed a clean-up strategy for profiling of multiple phytohormones, which can overcome the challenge of structural and chemical diversity. By using a one-step dispersive solid-phase extraction (DSPE) combined with UPLC-MS/MS, 54 phytohormones including auxins, ABA, SA, JA, GAs and CKs were simultaneously analyzed from a single rice sample extract. Using the developed method, we investigated the spatiotemporal distribution of phytohormones in rice. The profiling of various tissues of rice at different growth stages revealed the complexity of metabolic regulation and allocations of phytohormone species.

Conclusion: A rapid one-step method was developed for the simultaneous analysis of six groups of phytohormones, including cytokinins, auxins, salicylic acid, jasmonates, abscisic acid and gibberellins in a single run, using UPLCESI-MS/MS. The proposed method was successfully applied to investigate spatiotemporal distribution of multiple phytohormones in rice. The spatiotemporal information obtained may be helpful for better understanding of phytohormones functions throughout life cycle of rice when integrated into transcriptome and other omics data.

Keywords: UPLC-MS/MS, Phytohormones, Spatiotemporal distribution, Rice

\section{Background}

Phytohormones are a group of naturally occurring, organic substances which influence physiological processes at low concentrations [1]. They play crucial roles in almost all stages of plant growth and development, from embryogenesis to senescence. In addition, they also regulate response of plant to biotic and abiotic stress [2]. Phytohormones have been categorized into several groups based on their structures and physiological functions,

\footnotetext{
*Correspondence: yqfeng@whu.edu.cn

tWen-Jing Cai and Tian-Tian Ye contributed equally to this work Key Laboratory of Analytical Chemistry for Biology and Medicine (Ministry of Education), Department of Chemistry, Wuhan University, Wuhan 430072, People's Republic of China
}

including auxins, cytokinins (CKs), abscisic acid (ABA), jasmonates (JAs), salicylates, gibberellins (GAs), ethylene (ET), brassinosteroids (BRs), polyamines, signal peptides and the more-recently-discovered hormones, strigolactones (SLs) [1]. Each class of phytohormone has characteristic biological functions. However, increasing evidence shows that multiple phytohormones can mediate plant growth and development by additive, synergistic or antagonistic actions [3-7]. Phytohormone concentration and distribution are determinants of phytohormone action [8]. Therefore, studies on phytohormone functions and regulation networks primarily rely on sensitive and high-throughput methods for quantification of endogenous phytohormones in plants. Accurate and 
simultaneous determination of multiple phytohormones enabled us to better understand the physiological functions and the regulatory networks of phytohormones. Hirano et al. [9] presented the dynamic changes of each phytohormone during rice microspore/pollen (MS/ POL) development by analysis of endogenous levels of ABA, CKs, GAs and IAA combined with the transcriptome results in mature anther. According to spatial and temporal distribution of CKs and the related gene function assays, Rijavec et al. [10] found that CKs may perform highly contrasting roles in the filial endosperm and maternal tissues of developing seed in maize. Based on phytohormone profiling and RNA-seq analyses, Chao et al. [11] discovered the specific combination of phytohormones involved in bud differentiation and shoot growth at different time points. Hence, simultaneous profiling of multiple classes of hormones, especially integrated with the results of related gene expression profilings, is a powerful tool to reveal the mechanisms and interactions of phytohormones in different growth and development stages of plants [10].

There are two ways to get information about the concentrations of multiple phytohormones in plant samples. One is to divide the sample to multiple portions for independent analysis of multiple classes of phytohormones respectively [9]. However, this requires a large amount of plant sample, which cannot meet the increasing demand for analysis of limited amounts of plant samples, such as a tiny organ of a rice. The other way is to develop methods for simultaneous determination of multiple phytohormones in one sample. Simultaneous analysis of multiple phytohormones is challenging due to their structural and chemical diversity, and the low contents in plant samples, usually at the nanomolar level, as well as the complex plant matrix. Therefore, it's of great significance to design a feasible strategy for simultaneous analysis of multiple phytohormones. Great efforts have been made. Additional file 1: Table S1 presents a summary of representative analytical methods for simultaneous determination of multiple phytohormones. Multiple steps involving liquidliquid extractions or solid-phase extractions, as well as combinations of them have been used for the removal of the sample matrix and enrichment of multiple phytohormones [12-30]. Kojima et al. [12] developed a multi-step strategy for determination of 43 phytohormones including auxins, ABA, GAs and CKs. The phytohormones in rice were stepwise separated into several fractions by multiple solid-phase extraction (SPE). "MS-probe" bromocholine was used for derivatization of fractions containing auxin, ABA and gibberellins to increase the MS detection sensitivity. Subsequently, phytohormones in each fraction were, respectively, analyzed using UPLC-MS/MS [12].
Cao et al. [14] reported a method using liquid chromatography-triple quadrupole mass spectrometry (LC-MS/ MS) for the profiling and quantification of 43 phytohormones and their major metabolites, including auxins, abscisic acid, jasmonic acid, salicylic acid, cytokinins and gibberellins in a single sample extract purified by binary extraction using commercial polymer anion exchange resin (PAX) and polymer cation exchange resin (PCX), respectively. Liu et al. described a method for simultaneous analysis of 24 acidic and alkaline phytohormones, in which a binary SPE using Oasis MCX cartridges for cations and Oasis MAX cartridges for anions was employed for purification and enrichment of phytohormones. Alkaline and acidic phytohormones were eluted from different SPE cartridges, respectively. The two fractions of elution were combined for UPLC-MS/MS analysis [15]. Obviously, these multiple SPE strategies were tedious and time-consuming. One-step methods can be more efficient. Recently, Meulebroek et al. [26] developed a generic extraction protocol combining an UPLC-Orbitrap-MS detection method for analysis of eight phytohormones (GA3, ABA, IAA, JA, SA, Z, BA and BL) in both tomato fruit and leaf tissue. Crude plant extract was just passed through a $30 \mathrm{kDa}$ Amicon ${ }^{\circledR}$ Ultra centrifugal filter unit prior to LC-MS analysis. Pan et al. described a protocol for quantitative analysis of major phytohormones in crude plant extracts by high-performance liquid chromatography-mass spectrometry. Dichloromethane was used to extract and purify seven major classes phytohormones from plant extract before LC-MS analysis [28, 31]. Although these one-step sample preparation protocols are simple and fast, the matrix effect does exist and the efficiency of purification should be further improved. Cai et al. [19] developed a method to comprehensively profile phytohormones, including 8 cytokinins (CKs), indole3-acetic acid (IAA), abscisicacid (ABA), jasmonic acid (JA) and 10 gibberellins (GAs) by $\mathrm{Fe}_{3} \mathrm{O}_{4} @ \mathrm{TiO}_{2}$-based magnetic solid-phase extraction coupled with ultra-performance liquid chromatography-electrospray tandem mass spectrometry $\left(\mathrm{Fe}_{3} \mathrm{O}_{4} @ \mathrm{TiO}_{2}\right.$-based MSPE-UPLC$\mathrm{MS} / \mathrm{MS}$ ). Whereas, to date, these materials are not readily available in most laboratories.

In the current work, we have developed a clean-up strategy for profiling of multiple phytohormones, which can overcome the challenge of structural and chemical diversity. By using a one-step dispersive solid-phase extraction (DSPE) combined with UPLC-MS/MS, 54 phytohormones including auxins, ABA, SA, JA, GAs and CKs were simultaneously analyzed from a single rice sample extract. Using the developed method, we have investigated the spatiotemporal distribution of phytohormones in rice. 


\section{Results and discussion}

Method for rapid quantification of phytohormones

Graphitized carbon black (GCB) has been widely used in QuEChERS method for sample cleanup because it can remove chlorophyll through $\pi-\pi$ interaction with porphyrin ring of chlorophyll [32-34]. In this study, we choose GCB for cleanup of plant samples. The overall procedure for extraction and purification of phytohormones is summarized in Fig. 1. Acetonitrile is used to extract phytohormones from plant [29], and graphitized carbon black (GCB) sorbent is employed for dispersive solid-phase extraction (DSPE) cleanup. In order to obtain an optimal extraction efficiency, three parameters including the amount of GCB, water content of sampling solution and extraction time were optimized (Additional file 1: Fig. S1). As the amount of GCB increased, more amount of analytes were adsorbed and less remained in the supernatant, resulting in decreased recoveries. When sampling in ACN, analytes would be adsorbed by GCB via hydrophilic interaction, so addition of $\mathrm{H}_{2} \mathrm{O}$ could improve the recoveries. Finally, $10 \mathrm{mg}$ of GCB for DSPE, 80\% ACN (v/v) for sampling and 3 min for extraction were employed for further experiments. Under the optimized conditions, the absolute recoveries of 54 phytohormones spiked in $80 \% \mathrm{ACN}(\mathrm{v} / \mathrm{v})$ and plant sample matrix were investigated respectively by using the

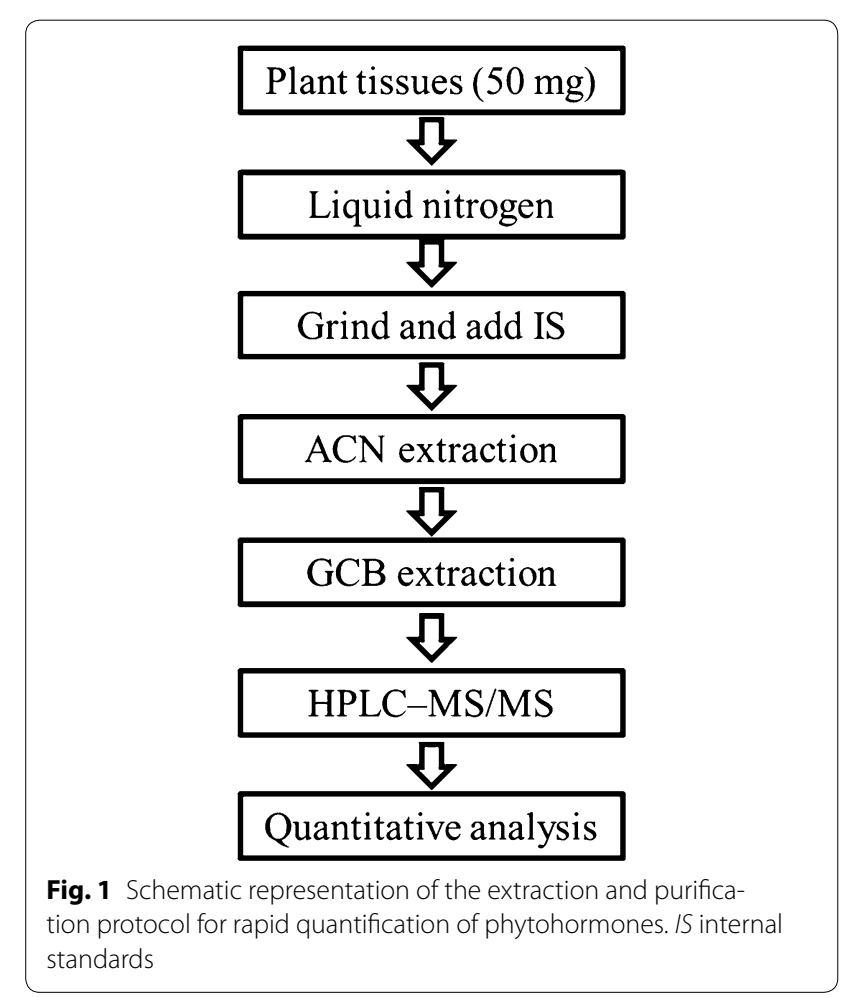

proposed DSPE. The recoveries in standards were calculated by comparing standards that were extracted with standards without extraction. The recoveries in matrix samples were calculated by comparing samples that were spiked and then extracted with those, which were extracted and then spiked. Internal standards were added to the samples before injection to UPLC-MS/MS to calibrate errors of instrument detection. And the results are listed in Additional file 1: Table S3. Recoveries of most phytohormones in sample matrix were higher than in standards. This might be because that the sample matrix may block GCB binding sites so that less phytohormones were absorbed to the GCB and more phytohormones remained in the supernatant, resulting in higher recoveries in sample matrix.

Ultra Performance Liquid Chromatography (UPLC) was employed for separation of 54 phytohormones. The chromatograms are shown in Fig. 2. Fifty-four tested analytes achieved baseline separation by UPLC, except for DZ7G and DZOG, MeStZ and MeScZ. Appropriate precursor to product ion transitions for each compound (54 molecular species) and their respective deuteriumlabeled internal standards were determined by UPLCESI-MS/MS (Additional file 1: Table S2). Cytokinins and auxins were detected in the positive ion mode, ABA, JA, $\mathrm{SA}$ and gibberellins were identified in the negative ion mode. A polarity-switching mode enables the analysis of compounds with different preferred ionization modes. In order to enhance the sensitivity of UPLC-ESI-MS/MS, six separate functions were implemented in the MRM mode so that only ions eluted during the specified retention windows were monitored. The reproducibility and accuracy of the proposed method were evaluated with intra-day and inter-day measurements. The intra-day precisions were obtained with extractions of five samples over a day, and the inter-day precisions were obtained by extracting samples in continuous three days. The RSDs of inter- and intra-day precision were below $11.8 \%$, and the relative recoveries were in the range of $80.3-120.4 \%$, indicating good reproducibility and accuracy of the method (Additional file 1: Table S5). The limits of quantifications (LOQs) were calculated as the signal-to-noise ratios of 10:1 on standards with 3 replicate injections, ranging from $0.05 \mathrm{fmol}$ for $2 \mathrm{MeStZ}$ to $29.92 \mathrm{fmol}$ for cZOG in cytokinins, from $0.18 \mathrm{fmol}$ for $\mathrm{GA}_{1}$ to $27.5 \mathrm{fmol}$ for $\mathrm{GA}_{9}$ in gibberellins, $12.88 \mathrm{fmol}$ for IAA, $93.29 \mathrm{fmol}$ for SA and 1.12 fmol for JA (Additional file 1: Table S4). The LOQs are comparable with the majority of the methods in Additional file 1: Table S1. However, the presented method has the advantage of being faster than most other methods and can analyze multiple phytohormones in a single UPLC-ESI-MS/MS run (Additional file 1: Table S1). 

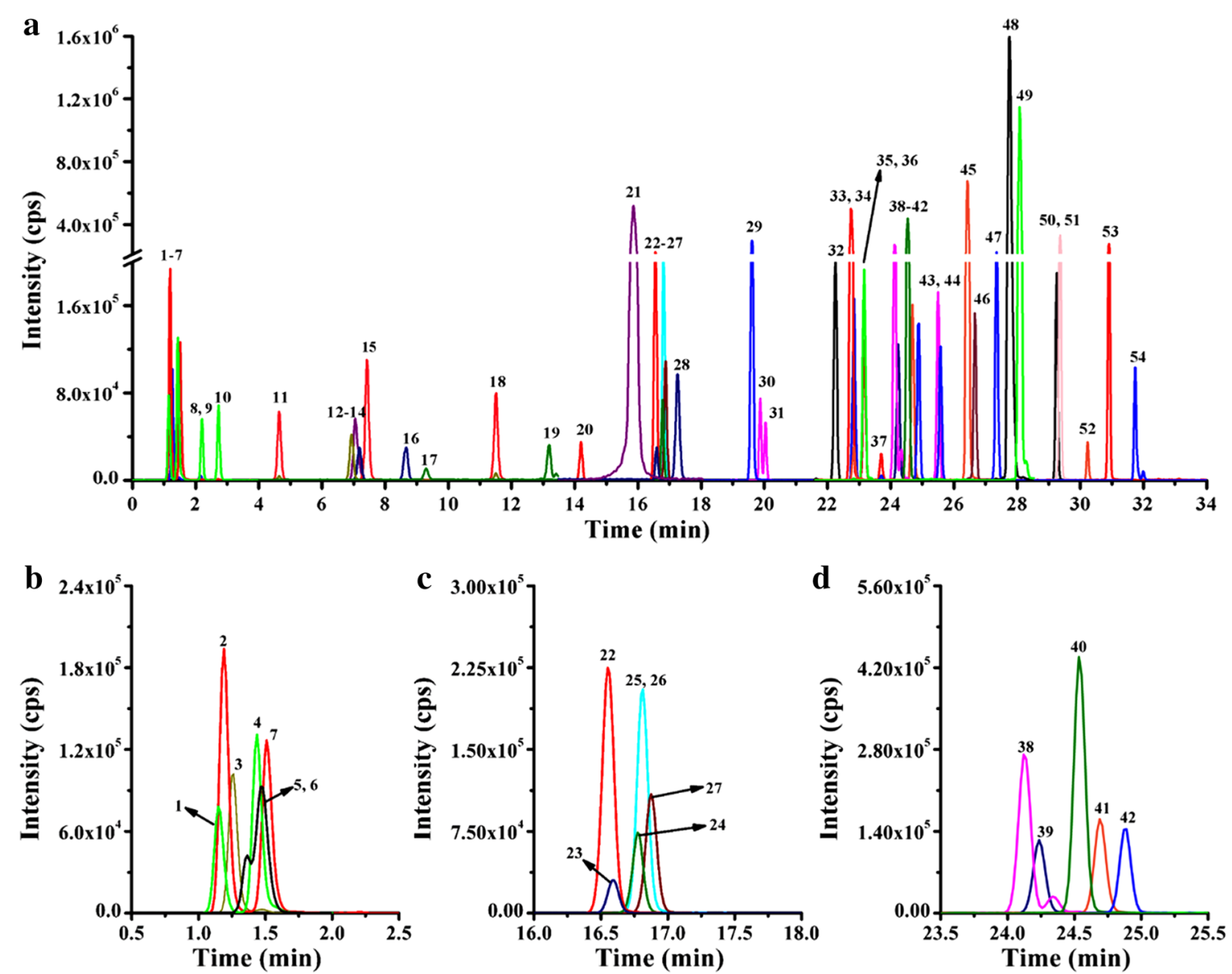

Fig. 2 The MRM chromatograms of 54 phytohormones analyzed by UPLC-ESI-MS/MS. a Peak 1-54; b peak 1-7; c peak 22-27; d peak 38-42; 1, tZ7G; 2, tZ; 3, DZ; 4, cZOG; 5, DZ7G; 6, DZOG; 7, cZ; 8, DZ9G; 9, tZ9G; 10, cZ9G; 11, iP7G; 12, iP; 13, DZR; 14, tZR; 15, GA; 16, cZR; 17, GA 29 ; 18, iP9G; 19, 12OHJA; 20, GA $23 ; 21$, SA; 22, 2CltZ; 23, GA $;$ 24, iPR; 25, 2MeStZ; 26, 2MeScZ; 27, GA ; 28, IAA; 29, GA ; 30, 2MeStZR; 31, 2MeStZR; 32, ABA; 33, GA 13 ; 34, $\mathrm{GA}_{5} ; 35, \mathrm{GA}_{19} ; 36, \mathrm{GA}_{20} ; 37, \mathrm{GA}_{44} ; 38, \mathrm{JA} ; 39$, IBA; 40, GA $34 ; 41$, 2MeSiP; 42, GA 51 ; 43, GA 53 ;4, 2MeSiPR; 45, GA $;$ 46, GA $; 47, \mathrm{GA}$ 24; 48, JA-leu; 49, JA-phe; $50, \mathrm{GA}_{15} ; 51, \mathrm{GA}_{9} ; 52,2 \mathrm{BSiP} ; 53, \mathrm{GA}_{12} ; 54$, OPDA. The full names and abbreviations of the phytohormones can be found in "Chemicals and reagents" section

\section{Spatiotemporal distribution of phytohormones in rice}

To evaluate the spatiotemporal distribution of phytohormone species in rice. Root and leaves of rice (cv. 'Zhenshan 97B') plants were harvested at seedling stage and tillering stage. Root, senescent leaves, frag leaf and ear were harvested at grain-filling stage and mature grain stage. Then the endogenous hormone contents were analyzed. Among the 54 phytohormones investigated, 36 were quantified, including $18 \mathrm{CK}$ species, 10 GA species, 5 JA species, IAA, ABA, and SA. The measurement results are shown in Additional file 1: Table S6. And the examples of chromatograms of rice tissue (rice ear at filling stage) are shown in Fig. 3. Accumulation of phytohormones displayed substantial variation in their abundance in different tissues of rice at different stages, as shown in the heat map (Fig. 4a). More species of hormones were detected in ear of rice at grain-filling stage than in other tissues, of which most showed higher concentrations. These indicated that phytohormones play important roles in seed development of rice [35].

In terms of cytokinins, cis-zeatin (cZ)-type cytokinins were dominant in all rice tissues investigated at all growth stages (Fig. 4b). The most abundant CK metabolite detected was cisZ-O-glucoside (cZOG) (Fig. 4a), being consistent with the previous reports [8, 36]. Glucosides were the major form of accumulated cytokinins in all tissues investigated, which are inactive and are thought to play a role in homeostasis of the hormones [1]. To better explain the dynamic change of endogenous levels of phytohormone species in rice, a metabolic pathway was shown in Fig. 5. In root, DZR was only detected 


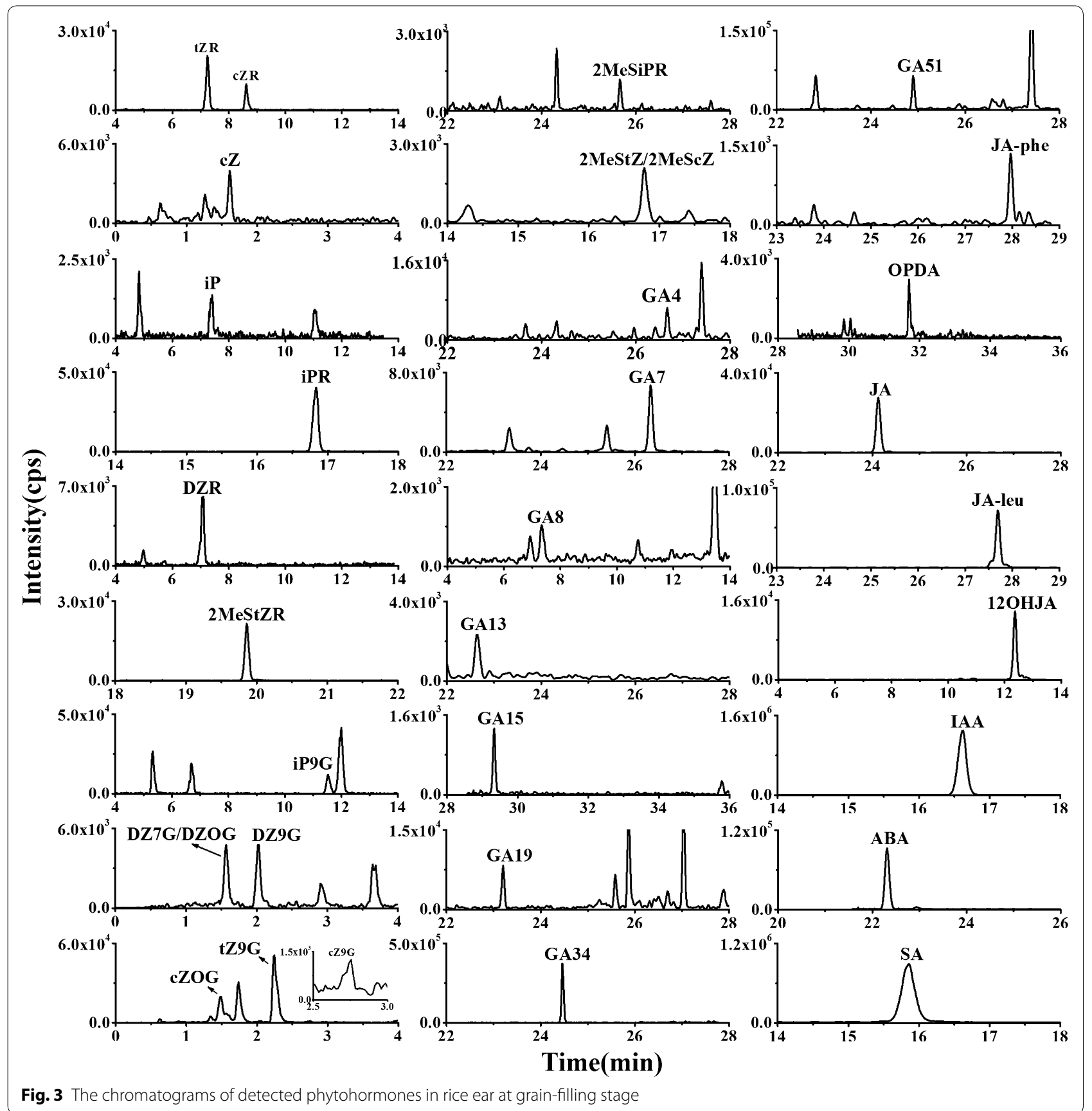

at tillering stage. Contents of iP9G increased as the plants grown up. tZ9G, cZ9G and DZ9G increased from seedling stage to filling stage, but declined at mature grain stage. In frag leaf, cytokinin precursors such as DZR and cZR accumulated in frag leaf at mature grain stage. However, glucosides decreased in frag leaf when the rice grown mature, except for tZ9G and cZOG. For ear, almost all the cytokinins investigated decreased in the mature ear, except for DZ.
As for gibberellins, distinct tissue-specific accumulation patterns were observed. $\mathrm{GA}_{7}, \mathrm{GA}_{51}$ and $\mathrm{GA}_{34}$ were mainly accumulated in ear. Bioactive $\mathrm{GA}_{4}$ was detected in most of the tissues investigated except for senescent leaves at filling stage and tissues at mature grain stage. $\mathrm{GA}_{7}$ was only detected in ear of rice at filling stage. For root, GA precursor $\mathrm{GA}_{53}$ and downstream $\mathrm{GA}_{51}$ accumulated in root at mature grain stage. Bioactive $\mathrm{GA}_{4}$ showed significant reduction in root when the rice grown mature. 


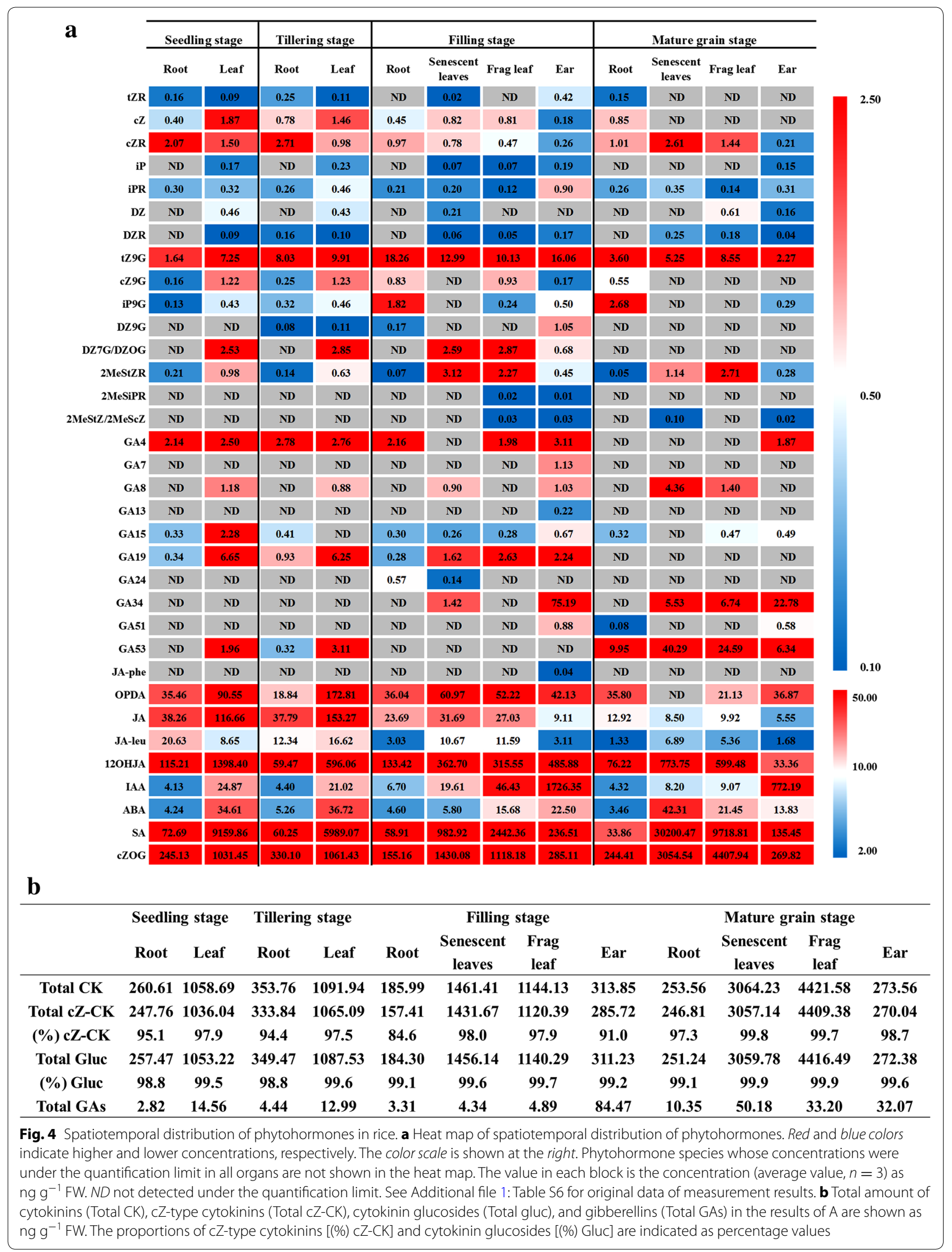




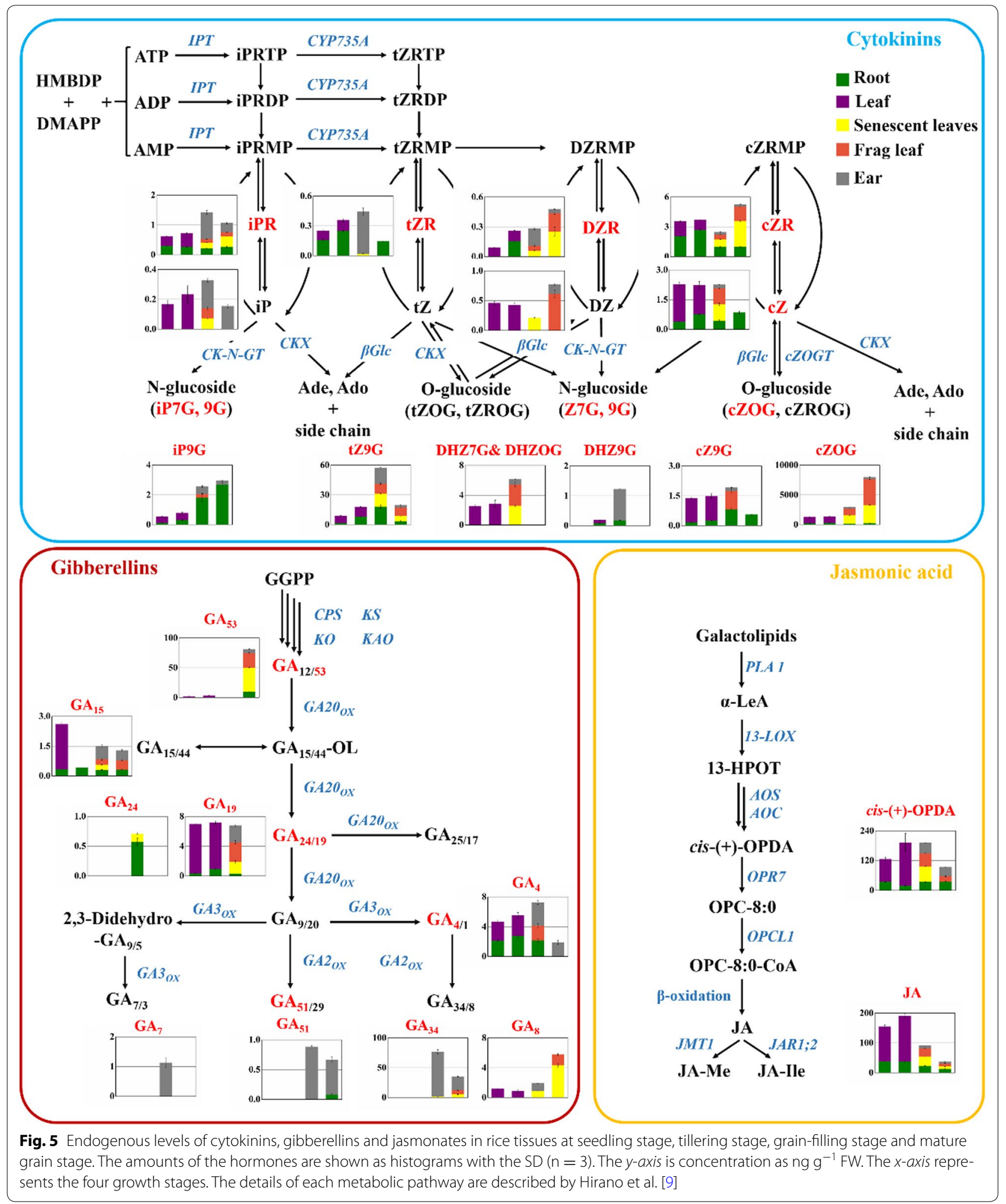


For frag leaf, GA precursor $\mathrm{GA}_{53}$ and downstream $\mathrm{GA}_{8}$ accumulated at mature grain stage. For ear, GA precursor $\mathrm{GA}_{53}$ accumulated in mature ear, while the downstream GAs decreased, including the bioactive $\mathrm{GA}_{4}$ and $\mathrm{GA}_{7}$, and the deactivated $\mathrm{GA}_{8}$. $\mathrm{GA}_{19}$ accumulated in ear at grain-filing stage, but decreased to a very low level in mature ear, being consistent with the result Suzuki reported [37]. The decrease in $\mathrm{GA}_{19}$ content at mature grain stage may indicate vigorous consumption of $\mathrm{GA}_{19}$, which acts as a pool GA in the biosynthetic pathway to $\mathrm{GA}_{7}, \mathrm{GA}_{51}, \mathrm{GA}_{34}$ and $\mathrm{GA}_{8}$, which highly accumulated in ear at grain-filing stage.

The rice tissues also contained large amounts of ABA, IAA, OPDA, JA and SA. ABA showed higher concentrations in leaf than in root at seedling stage and tillering stage, and the content kept steady. From grain-filling stage to mature grain stage, ABA increased in senescent leaves, but did not change in the other tissues. IAA showed the lowest accumulation in root. However, an extremely high accumulation in ear was observed. The high accumulation of IAA in ear is consistent with the highly expressed genes related to auxin biosynthetic and metabolic processes, polar auxin transport, homeostasis and auxin-mediated signaling [35]. Concentration of JA declined in all tissues as the plants grown mature. Taken together, these results indicate that our analysis could show the spatiotemporal distribution pattern of the phytohormones in rice, and that the phytohormones are differentially distributed in rice tissues at different growth stages. However, for the further understanding of phytohormone function, some important clues obtained by transcriptome and other omics are needed.

\section{Conclusions}

In this study, we have developed a rapid one-step method for the simultaneous analysis of six groups of phytohormones, including cytokinins, auxins, salicylic acid, jasmonates, abscisic acid and gibberellins in a single run, using UPLC-ESI-MS/MS. The proposed method was successfully applied to investigate spatiotemporal distribution of multiple phytohormones in rice. The spatiotemporal information obtained may be helpful for better understanding of phytohormones functions throughout life cycle of rice when integrated into transcriptome and other omics data.

\section{Methods}

\section{Chemicals and reagents}

Phytohormones standards: indole-3-acetic acid (IAA), indole-3-butyric acid (IBA), abscisic acid (ABA), salicylic acid (SA), jasmonic acid (JA), 2H-jasmonic acid (2H-JA) Jasmonic acid-leucine (JA-Ieu), Jasmonic acid-phenylalanine (JA-phe), 12-OH-jasmonic acid (12-OH-JA), 12-oxophytodienoic acid (OPDA), gibberellins ( $\mathrm{GA}_{1}$, $\mathrm{GA}_{3}, \mathrm{GA}_{4}, \mathrm{GA}_{5}, \mathrm{GA}_{6}, \mathrm{GA}_{7}, \mathrm{GA}_{8}, \mathrm{GA}_{9}, \mathrm{GA}_{12}, \mathrm{GA}_{13}, \mathrm{GA}_{15}$, $\left.\mathrm{GA}_{19}, \mathrm{GA}_{20}, \mathrm{GA}_{23}, \mathrm{GA}_{24}, \mathrm{GA}_{29}, \mathrm{GA}_{34}, \mathrm{GA}_{44}, \mathrm{GA}_{51}, \mathrm{GA}_{53}\right)$; trans-zeatin (tZ), cis-zeatin (cZ), transzeatin-7-glucoside (tZ7G), trans-zeatin-9-glucoside (tZ9G), cis-zeatin9-glucoside (cZ9G), cis-zeatin-O-glucoside (cZOG), dihydrozeatin (DZ), dihydrozeatin-7-glucoside (DZ7G), dihydrozeatin-9-glucoside (DZ9G), dihydrozeatin-Oglucoside (DZOG), isopentenyladenine (iP), $\mathrm{N}^{6}$-isopentenyladenine-7-glucoside (iP7G), $\mathrm{N}^{6}$-isopentenyladenine 9-glucoside (iP9G), trans-zeatin-riboside (tZR), cisriboside (cZR), dihydrozeatin riboside (DZR), isopentenyladenine riboside (iPR), 2-chloro-trans- zeatin (2CltZ), 2-methylthio-trans-zeatin (2MeStZ), 2-methylthio-cis-zeatin (2MeScZ), 2-methylthio-trans-zeatinriboside (2MeStZR), 2-methylthio-cis-zeatin-riboside (2MeScZR), 2-methylthio- $\mathrm{N}^{6}$-isopentenyladenine (2MeSiP), 2-methylthio- $\mathrm{N}^{6}$ - isopentenyladenine riboside (2MeSiPR), 2-benzylthio- $\mathrm{N}^{6}$-isopentenyladenine (2BSiP) and stable isotope-labeled standards: $\left[{ }^{2} \mathrm{H}_{2}\right] \mathrm{IAA}$, $\left[{ }^{2} \mathrm{H}_{6}\right] \mathrm{ABA}, \quad\left[{ }^{2} \mathrm{H}_{4}\right] \mathrm{SA}, \quad\left[{ }^{2} \mathrm{H}_{2}\right] \mathrm{GA}_{1}, \quad\left[{ }^{2} \mathrm{H}_{2}\right] \mathrm{GA}_{4}, \quad\left[{ }^{2} \mathrm{H}_{2}\right] \mathrm{GA}_{5}$, $\left[{ }^{2} \mathrm{H}_{2}\right] \mathrm{GA}_{6}, \quad\left[{ }^{2} \mathrm{H}_{2}\right] \mathrm{GA}_{7}, \quad\left[{ }^{2} \mathrm{H}_{2}\right] \mathrm{GA}_{8}, \quad\left[{ }^{2} \mathrm{H}_{2}\right] \mathrm{GA}_{9}, \quad\left[{ }^{2} \mathrm{H}_{2}\right] \mathrm{GA}_{12}$, $\left[{ }^{2} \mathrm{H}_{2}\right] \mathrm{GA}_{15},\left[{ }^{2} \mathrm{H}_{2}\right] \mathrm{GA}_{20},\left[{ }^{2} \mathrm{H}_{2}\right] \mathrm{GA}_{24},\left[{ }^{2} \mathrm{H}_{2}\right] \mathrm{GA}_{34},\left[{ }^{2} \mathrm{H}_{2}\right] \mathrm{GA}_{44}$, $\left[{ }^{2} \mathrm{H}_{2}\right] \mathrm{GA}_{51}, \quad\left[{ }^{2} \mathrm{H}_{2}\right] \mathrm{GA}_{53}, \quad\left[{ }^{2} \mathrm{H}_{5}\right] \mathrm{tZ}, \quad\left[{ }^{15} \mathrm{~N}_{4}\right] \mathrm{cZ}, \quad\left[{ }^{2} \mathrm{H}_{5}\right] \mathrm{tZ} 7 \mathrm{G}$, $\left[{ }^{2} \mathrm{H}_{5}\right]$ tZ9G, $\left[{ }^{2} \mathrm{H}_{3}\right] \mathrm{DZ},\left[{ }^{2} \mathrm{H}_{5}\right]$ DZ9G, $\left[{ }^{2} \mathrm{H}_{7}\right]$ DZOG, $\left[{ }^{2} \mathrm{H}_{6}\right] \mathrm{iP}$, $\left[{ }^{2} \mathrm{H}_{6}\right]$ iP9G, $\left[{ }^{2} \mathrm{H}_{5}\right] \mathrm{tZR},\left[{ }^{2} \mathrm{H}_{3}\right]$ DZR, $\left[{ }^{2} \mathrm{H}_{6}\right]$ iPR were all purchased from Olchemim Ltd. (Olomouc, Czech Republic). Acetonitrile (ACN, HPLC grade) was obtained from Tedia Co. (Fairfield, OH, USA). Ultra-pure water used throughout the study was purified with Milli-Q system (Milford, MA, USA). Formic acid (FA, 88\%) was purchased from Sinopharm Chemical Reagent (Shanghai, China). Graphitized carbon black (GCB) was purchased from BOSHI Biotechnology Co., Ltd (shanghai, china, http://www.boshibio.com.cn).

\section{Plant materials}

Rice (Oryza sativa ssp. indica cv. Zhenshan 97B) (kindly provided by Dr. Qian Qian from State Key Laboratory of Rice Biology, China National Rice Research Institute) plants were grown under natural field conditions during the rice-growing season (from June to October). Root and leaves were harvested at seedling stage and late-tillering stage. Root, senescent leaves, frag leaf and ear were harvested at grain-filling stage and mature grain stage. All the samples were harvested at 10:00-12:00 h, placed in liquid nitrogen immediately, and stored at $-80{ }^{\circ} \mathrm{C}$. Samples were taken from three different plants per line for three biological replicates.

\section{Sample pretreatment}

As shown in Fig. 2, plant tissues (root, leaf and ear) (50 mg FW) were frozen with liquid nitrogen, grounded 
into powder with liquid nitrogen and then transferred into a $1.5-\mathrm{mL}$ centrifuge tube. $\left[{ }^{2} \mathrm{H}_{2}\right]$ IAA $(1.0 \mathrm{ng}),\left[{ }^{2} \mathrm{H}_{6}\right]$ ABA (1.0 ng), 2H-JA (1.0 ng) $\left[{ }^{2} \mathrm{H}_{4}\right] \mathrm{SA}(50 \mathrm{ng}),\left[{ }^{2} \mathrm{H}_{2}\right] \mathrm{GA}_{1}$ (0.5 ng), $\left[{ }^{2} \mathrm{H}_{2}\right] \mathrm{GA}_{4}(0.5 \mathrm{ng}),\left[{ }^{2} \mathrm{H}_{2}\right] \mathrm{GA}_{5}(0.5 \mathrm{ng}),\left[{ }^{2} \mathrm{H}_{2}\right] \mathrm{GA}_{6}$ (0.5 ng), $\left[{ }^{2} \mathrm{H}_{2}\right] \mathrm{GA}_{7}(0.5 \mathrm{ng}),\left[{ }^{2} \mathrm{H}_{2}\right] \mathrm{GA}_{8}(0.5 \mathrm{ng}),\left[{ }^{2} \mathrm{H}_{2}\right] \mathrm{GA}_{9}$ (0.5 ng), $\left[{ }^{2} \mathrm{H}_{2}\right] \mathrm{GA}_{12}(0.5 \mathrm{ng}),\left[{ }^{2} \mathrm{H}_{2}\right] \mathrm{GA}_{15}(0.5 \mathrm{ng}),\left[{ }^{2} \mathrm{H}_{2}\right]$ $\mathrm{GA}_{20}(0.5 \mathrm{ng}),\left[{ }^{2} \mathrm{H}_{2}\right] \mathrm{GA}_{24}(0.5 \mathrm{ng}),\left[{ }^{2} \mathrm{H}_{2}\right] \mathrm{GA}_{34}(0.5 \mathrm{ng})$, $\left[{ }^{2} \mathrm{H}_{2}\right] \mathrm{GA}_{44} \quad(0.5 \mathrm{ng}), \quad\left[{ }^{2} \mathrm{H}_{2}\right] \mathrm{GA}_{51} \quad(0.5 \mathrm{ng}), \quad\left[{ }^{2} \mathrm{H}_{2}\right] \mathrm{GA}_{53}$ $(0.5 \mathrm{ng}),\left[{ }^{2} \mathrm{H}_{5}\right] \mathrm{tZ}(0.1 \mathrm{ng}),{ }^{15} \mathrm{~N}_{4}$-cZ $(0.1 \mathrm{ng}),\left[{ }^{2} \mathrm{H}_{5}\right] \mathrm{tZ} 7 \mathrm{G}$ $(0.1 \mathrm{ng}),\left[{ }^{2} \mathrm{H}_{5}\right]$ tZ9G $(0.1 \mathrm{ng}),\left[{ }^{2} \mathrm{H}_{3}\right] \mathrm{DZ}(0.1 \mathrm{ng}),\left[{ }^{2} \mathrm{H}_{5}\right] \mathrm{DZ} 9 \mathrm{G}$ $(0.1 \mathrm{ng}),\left[{ }^{2} \mathrm{H}_{7}\right]$ DZOG $(0.1 \mathrm{ng}),\left[{ }^{2} \mathrm{H}_{6}\right] \mathrm{iP}(0.1 \mathrm{ng}),\left[{ }^{2} \mathrm{H}_{6}\right] \mathrm{iP9G}$ (0.1 ng), $\left[{ }^{2} \mathrm{H}_{5}\right] \mathrm{tZR}(0.1 \mathrm{ng}),\left[{ }^{2} \mathrm{H}_{3}\right]$ DZR (0.1 ng), $\left[{ }^{2} \mathrm{H}_{6}\right] \mathrm{iPR}$ $(0.1 \mathrm{ng})$ mixture (in $5 \mu \mathrm{L} \mathrm{ACN})$ was quickly added to the samples to serve as internal standards (I.S.) for the quantification. Then ACN $(0.5 \mathrm{~mL})$ was added and the mixture was vortexed for $30 \mathrm{~s}$. After standing at $-20^{\circ} \mathrm{C}$ for $12 \mathrm{~h}$, the supernatant was collected upon centrifugation at $10,000 \times g$ under $4{ }^{\circ} \mathrm{C}$ for $20 \mathrm{~min}$. Subsequently, the supernatant was evaporated to dryness under a mild nitrogen stream at $35^{\circ} \mathrm{C}$ and redissolved in $0.5 \mathrm{~mL} \mathrm{ACN}$ containing $80 \% \mathrm{ACN}(\mathrm{v} / \mathrm{v})$. The sample solution was added into a 1.5 -mLvial containing $10 \mathrm{mg}$ graphitized carbon black. The mixture was vortexed vigorously for $3 \mathrm{~min}$ and the supernatant was transported to a $1.5-\mathrm{mL}$ vial followed by evaporating to dryness. The residues were redissolved in $5 \% \mathrm{ACN}(\mathrm{v} / \mathrm{v})(50 \mu \mathrm{L})$ and $10 \mu \mathrm{L}$ was injected for UPLC$\mathrm{MS} / \mathrm{MS}$ analysis.

\section{Instruments and analytical conditions}

Analysis of phytohormones was performed on a UPLCESI (+/-)-MS/MS system consisting of a AB SCIEX 4500 triple quadrupole mass spectrometer (Foster City, CA, USA) with an electrospray ionization source (Turbo Ionspray), a Shimadzu LC-30AD.

HPLC system (Tokyo, Japan) with two 30AD pumps, a SIL-30AC auto sampler, a CTO-30A thermostat column compartment, and a DGU-20A5R degasser. Data acquisition and processing were performed using $\mathrm{AB}$ SCIEX Analyst 1.6 software (Foster City, CA, USA).

The HPLC separation was performed on a on a Shimpack XR-ODS $\amalg$ column $(75 \mathrm{~mm} \times 2.0 \mathrm{~mm}$ i.d., $1.6 \mu \mathrm{m})$ purchased from Shimadzu (Tokyo, Japan) at $40{ }^{\circ} \mathrm{C}$. A 52 -min gradient of $0.1 \%$ FA (A) and ACN (B) was employed for the separation with a flow rate of $0.4 \mathrm{~mL} /$ min. A gradient programme of $4 \mathrm{~min} 5-5 \% \mathrm{~B}, 6 \mathrm{~min}$ $5-7 \%$ B, 10 min $7-20 \%$ B, 20 min $20-80 \%$ B, 2 min $80-5 \%$ and 5 min $5 \%$ B was used.

Multiple reaction monitoring (MRM) and the appropriate product ions were chosen to quantify phytohormones (Additional file 1: Table S2). The optimized conditions of MRM experiments were as follows: curtain gas, $40 \mathrm{psi}$; ion spray voltage, $5000 \mathrm{~V}$ for positive ion mode and $-4500 \mathrm{~V}$ for negative ion mode; turbo heater temperature (TEM), $500{ }^{\circ} \mathrm{C}$; nebulizing gas (Gas 1), 55 psi; heated gas (Gas 2), 40 psi. Data acquisition, peak integration, and the calculations were performed using Analyst 1.6.1 software (AB Sciex).

\section{Additional file}

Additional file 1: Figure S1. Investigation of DSPE conditions. Table S1. Representative analytical methods for simultaneous determination of multiple phytohormones. Table S2. Summary of precursor-to-product ion transitions used for the quantification of phytohormones using UPLC-ESI-MS/MS. Table S3. The absolute recoveries of phytohormones extracted by GCB. Table S4. Linearities, LODs and LOQs of 54 phytohormones by GCB-based MSPE-UPLC-MS/MS method. Table S5. Precisions (intra- and inter-day) and recoveries of 54 phytohormones by GCB-based MSPE-UPLC-MS/MS method. Table S6. Contents of detected endogenous phytohormones in rice tissues.

\section{Authors' contributions}

YQF designed and supervised this study. WJC and QW performed the data analysis. LY, WJC, TTY and QFZ contributed to materials collection. YQF, WJC and TTY prepared the manuscript, and all the authors critically read and approved the manuscript.

\section{Acknowledgements}

We thank Dr. Qian Qian for providing the rice seed; Dr Yang-Sheng Li for help with the planting; Lei Yu, Quan-Fei Zhu, and Shu-Jian Zheng for their help with sample preparation and plant care. We are also grateful to the reviewers for their helpful suggestions. This work was supported by the National Natural Science Foundation of China $(21475098,91217309)$, and the Natural Science Foundation of Hubei Province, China (2014CFA002).

\section{Competing interests}

The authors declare that they have no competing interests.

\section{Availability of data and material}

All data generated or analysed during this study are included in this published article [and its supplementary information files].

\section{Funding}

This work was supported by the National Natural Science Foundation of China (21475098, 91217309), and the Natural Science Foundation of Hubei Province, China (2014CFA002).

Received: 20 August 2016 Accepted: 3 November 2016 Published online: 17 November 2016

References

1. Davies PJ. The plant hormones: their nature, occurrence, and functions. In: Davies PJ, editor. Plant hormones: biosynthesis, signal transduction, action! Dordrecht: Springer. p. 1-15.

2. Kumar PP. Regulation of biotic and abiotic stress responses by plant hormones. Plant Cell Rep. 2013;32:943.

3. Voesenek LACJ, Benschop JJ, Bou J, Cox MCH, Groeneveld HW, Millenaar FF, Vreeburg RAM, Peeters AJM. Interactions between plant hormones regulate submergence-induced shoot elongation in the flooding-tolerant dicot Rumex palustris. Ann Bot. 2003;91:205-11.

4. Nemhauser JL, Hong FX, Chory J. Different plant hormones regulate similar processes through largely nonoverlapping transcriptional responses. Cell. 2006;126:467-75.

5. Thao NP, Khan MIR, Thu NBA, Hoang XLT, Asgher M, Khan NA, Tran LSP. Role of ethylene and its cross talk with other signaling molecules in plant responses to heavy metal stress. Plant Physiol. 2015;169:73-84. 
6. Kuppusamy KT, Walcher CL, Nemhauser JL. Cross-regulatory mechanisms in hormone signaling. Plant Mol Biol. 2009;69:375.

7. Jiang CJ, Shimono M, Sugano S, Kojima M, Yazawa K, Yoshida R, Inoue H, Hayashi N, Sakakibara H, Takatsuji H. Abscisic acid interacts antagonistically with salicylic acid signaling pathway in rice-Magnaporthe grisea interaction. Mol Plant Microbe Interact. 2010;23:791-8.

8. Kudo T, Akiyama K, Kojima M, Makita N, Sakurai T, Sakakibara H. UniVIO: a multiple omics database with hormonome and transcriptome data from rice. Plant Cell Physiol. 2013;54:e9.

9. Hirano K, Aya K, Hobo T, Sakakibara H, Kojima M, Shim RA, Hasegawa Y, Ueguchi-Tanaka M, Matsuoka M. Comprehensive transcriptome analysis of phytohormone biosynthesis and signaling genes in microspore/pollen and tapetum of rice. Plant Cell Physiol. 2008;49:1429-50.

10. Rijavec T, Jain M, Dermastia M, Chourey PS. Spatial and temporal profiles of cytokinin biosynthesis and accumulation in developing caryopses of maize. Ann Bot. 2011;107:1235-45.

11. Chao WS, Dogramaci M, Horvath DP, Anderson JV, Foley ME. Phytohormone balance and stress-related cellular responses are involved in the transition from bud to shoot growth in leafy spurge. BMC Plant Biol. 2016;16:47.

12. Kojima M, Kamada-Nobusada T, Komatsu H, Takei K, Kuroha T, Mizutani M, Ashikari M, Ueguchi-Tanaka M, Matsuoka M, Suzuki K, Sakakibara H. Highly sensitive and high-throughput analysis of plant hormones using MS-probe modification and liquid chromatography-tandem mass spectrometry: an application for hormone profiling in Oryza sativa. Plant Cell Physiol. 2009;50:1201-14.

13. Fan SF, Wang XP, Li PW, Zhang Q, Zhang W. Simultaneous determination of 13 phytohormones in oilseed rape tissues by liquid chromatographyelectrospray tandem mass spectrometry and the evaluation of the matrix effect. J Sep Sci. 2011;34:640-50.

14. Cao ZY, Sun LH, Mou RX, Zhang LP, Lin XY, Zhu ZW, Chen MX. (PAX\&PCX) Profiling of phytohormones and their major metabolites in rice using binary solid-phase extraction and liquid chromatography-triple quadrupole mass spectrometry. J Chromatogr A. 2016;1451:67-74.

15. Liu S, Chen W, Qu L, Gai Y, Jiang X. Simultaneous determination of 24 or more acidic and alkaline phytohormones in femtomole quantities of plant tissues by high-performance liquid chromatography-electrospray ionization-ion trap mass spectrometry. Anal Bioanal Chem. 2013;405:1257-66.

16. Niu Q, Zong Y, Qian M, Yang F, Teng Y. Simultaneous quantitative determination of major plant hormones in pear flowers and fruit by UPLC/ ESI-MS/MS. Anal Methods. 2014;6:1766-73.

17. Gouthu S, Morre J, Maier CS, Deluc LG. An analytical method to quantify three plant hormone families in grape berry using liquid chromatography and multiple reaction monitoring mass spectrometry. In: Gang RD, editor. Phytochemicals, plant growth, and the environment. New York, NY: Springer New York. 2013. p. 19-36.

18. Dobrev PI, Kaminek M. Fast and efficient separation of cytokinins from auxin and abscisic acid and their purification using mixed-mode solidphase extraction. J Chromatogr A. 2002;950:21-9.

19. Cai BD, Yin J, Hao YH, Li YN, Yuan BF, Feng YQ. Profiling of phytohormones in rice under elevated cadmium concentration levels by magnetic solidphase extraction coupled with liquid chromatography tandem mass spectrometry. J Chromatogr A. 2015;1406:78-86.

20. Ma Z, Ge L, Lee AS, Yong JW, Tan SN, Ong ES. Simultaneous analysis of different classes of phytohormones in coconut (Cocos nucifera L.) water using high-performance liquid chromatography and liquid chromatography-tandem mass spectrometry after solid-phase extraction. Anal Chim Acta. 2008;610:274-81.

21. Izumi Y, Okazawa A, Bamba T, Kobayashi A, Fukusaki E. Development of a method for comprehensive and quantitative analysis of plant hormones by highly sensitive nanoflow liquid chromatography-electrospray ionization-ion trap mass spectrometry. Anal Chim Acta. 2009;648:215-25.
22. Wang X, Zhao P, Liu X, Chen J, Xu J, Chen H, Yan X. Quantitative profiling method for phytohormones and betaines in algae by liquid chromatography electrospray ionization tandem mass spectrometry. Biomed Chromatogr. 2014;28:275-80.

23. Flokova K, Tarkowska D, Miersch O, Strnad M, Wasternack C, Novak O. UHPLC-MS/MS based target profiling of stress-induced phytohormones. Phytochemistry. 2014;105:147-57.

24. Chiwocha SDS, Abrams SR, Ambrose SJ, Cutler AJ, Loewen M, Ross ARS, Kermode AR. A method for profiling classes of plant hormones and their metabolites using liquid chromatography-electrospray ionization tandem mass spectrometry: an analysis of hormone regulation of thermodormancy of lettuce (Lactuca sativa L.) seeds. Plant J. 2003; 35:405-17.

25. Kasote DM, Ghosh R, Chung JY, Kim J, Bae I, Bae H. Multiple reaction monitoring mode based liquid chromatography-mass spectrometry method for simultaneous quantification of brassinolide and other plant hormones involved in abiotic stresses. Int J Anal Chem. 2016;2016:7214087.

26. Van Meulebroek L, Bussche JV, Steppe K, Vanhaecke L. Ultra-high performance liquid chromatography coupled to high resolution Orbitrap mass spectrometry for metabolomic profiling of the endogenous phytohormonal status of the tomato plant. J Chromatogr A. 2012;1260:67-80.

27. Müller M, Munné-Bosc S. Rapid and sensitive hormonal profiling of complex plant samples by liquid chromatography coupled to electrospray ionization tandem mass spectrometry. Plant Methods. 2011;7:37.

28. Pan $\mathrm{XQ}$, Welti $\mathrm{R}$, Wang $\mathrm{XM}$. Quantitative analysis of major plant hormones in crude plant extracts by high-performance liquid chromatographymass spectrometry. Nat Protoc. 2010;5:986-92.

29. Cai BD, Ye EC, Yuan BF, Feng YQ. Sequential solvent induced phase transition extraction for profiling of endogenous phytohormones in plants by liquid chromatography-mass spectrometry. J Chromatogr B Analyt Technol Biomed Life Sci. 2015;1004:23-9.

30. Castillo G, Torrecillas A, Nogueiras C, Michelena G, Sanchez-Bravo J, Acosta M. Simultaneous quantification of phytohormones in fermentation extracts of Botryodiplodia theobromae by liquid chromatographyelectrospray tandem mass spectrometry. World J Microbiol Biotechnol. 2014;30:1937-46.

31. Pan $X Q$, Welti $R$, Wang $X M$. Simultaneous quantification of major phytohormones and related compounds in crude plant extracts by liquid chromatography-electrospray tandem mass spectrometry. Phytochemistry. 2008;69:1773-81.

32. Lehotay SJ, Son KA, Kwon H, Koesukwiwat U, Fu W, Mastovska K, Hoh E, Leepipatpiboon N. Comparison of QuEChERS sample preparation methods for the analysis of pesticide residues in fruits and vegetables. J Chromatogr A. 2010;1217:2548-60.

33. Zheng HB, Zhao Q, Mo JZ, Huang YQ, Luo YB, Yu QW, Feng YQ. Quick, easy, cheap, effective, rugged and safe method with magnetic graphitized carbon black and primary secondary amine as adsorbent and its application in pesticide residue analysis. J Chromatogr A. 2013;1300:127-33.

34. Zhao $P$, Wang $L$, Jiang $Y$, Zhang $F$, Pan C. Dispersive cleanup of acetonitrile extracts of tea samples by mixed multiwalled carbon nanotubes, primary secondary amine, and graphitized carbon black sorbents. J Agric Food Chem. 2012;60:4026-33.

35. Xue LJ, Zhang JJ, Xue HW. Genome-wide analysis of the complex transcriptional networks of rice developing seeds. PLoS ONE. 2012;7:e31081.

36. Gajdosova S, Spichal L, Kaminek M, Hoyerova K, Novak O, Dobrev PI, Galuszka P, Klima P, Gaudinova A, Zizkova E, Hanus J, Dancak M, Travnicek B, Pesek B, Krupicka M, Vankova R, Strnad M, Motyka V. Distribution, biological activities, metabolism, and the conceivable function of ciszeatin-type cytokinins in plants. J Exp Bot. 2011;62:2827-40.

37. Suzuki Y, Kurogochi S, Murofushi N, Ota Y, Takahashi N. Seasonal changes of GA1, GA19 and abscisic acid in three rice cultivars. Plant Cell Physiol. 1981;22:1085-93. 